\title{
Attentional Modulation of Alpha/Beta and Gamma Oscillations Reflect Functionally Distinct Processes
}

\author{
Markus Bauer, ${ }^{1,2}$ Max-Philipp Stenner, ${ }^{1,3}$ Karl J. Friston, ${ }^{1}$ and Raymond J. Dolan ${ }^{1}$ \\ ${ }^{1}$ Wellcome Trust Centre for Neuroimaging, University College London, London WC1N 3BG, United Kingdom, ${ }^{2}$ School of Psychology, University of \\ Nottingham, Nottingham NG7 2RD, United Kingdom, and 32Department of Neurology, Otto-von-Guericke-University Magdeburg, 39120 Magdeburg, Germany
}

The brain adapts to dynamic environments by adjusting the attentional gain or precision afforded to salient and predictable sensory input. Previous research suggests that this involves the regulation of cortical excitability (reflected in prestimulus alpha oscillations) before stimulus onset that modulates subsequent stimulus processing (reflected in stimulus-bound gamma oscillations). We present two spatial attention experiments in humans, where we first replicate the classic finding of prestimulus attentional alpha modulation and poststimulus gamma modulation. In the second experiment, the task-relevant target was a stimulus change that occurred after stimulus onset. This enabled us to show that attentional alpha modulation reflects the predictability (precision) of an upcoming sensory target, rather than an attenuation of alpha activity induced by neuronal excitation related to stimulus onset. In particular, we show that the strength of attentional alpha modulations increases with the predictability of the anticipated sensory target, regardless of current afferent drive. By contrast, we show that the poststimulus attentional gamma enhancement is stimulus-bound and decreases when the subsequent target becomes more predictable. Hence, this pattern suggests that the strength of gamma oscillations is not merely a function of cortical excitability, but also depends on the relative mismatch of predictions and sensory evidence. Together, these findings support recent theoretical proposals for distinct roles of alpha/beta and gamma oscillations in hierarchical perceptual inference and predictive coding.

Key words: alpha; attention; gamma; magnetoencephalography; oscillations; predictive coding

\section{Introduction}

A long tradition of research in cognitive neuroscience has addressed the question of the extent to which our brain processes the sensory environment selectively and the mechanisms by which this selection occurs (Luck et al., 2000; Carrasco, 2011). Neurophysiological correlates of attentional modulation in prestimulus alpha/beta and poststimulus gamma oscillations have supported more traditional findings of early and late attentional selection (for review, see Luck et al., 2000). More recent theoretical proposals have considered the nature of attentional selection from the viewpoint of predictive coding and formulate attention in terms of Bayesian inference with the idea that expectations about the salience or precision of sensory information shape perception (Friston, 2005; Feldman and Friston, 2010).

A distinct alpha modulation in the prestimulus period (Worden et al., 2000; Fu et al., 2001; Bauer et al., 2012b; Mazaheri et al., 2014) and gamma modulation in the poststimulus period

Received Aug. 13, 2013; revised Sept. 4, 2014; accepted 0ct. 12, 2014.

Author contributions: M.B., K.J.F., and R.J.D. designed research; M.B. performed research; M.B. and M.-P.S. analyzed data; M.B., M.-P.S., K.J.F., and R.J.D. wrote the paper.

This work was funded by Wellcome Trust Grant 087756/Z/08/Z. This work was also supported by Wellcome Trust Senior Investigator Award 098362/Z/12/Z (to R.J.D.), and the Wellcome Trust Centre for Neuroimaging is supported by Core Funding Grant 091593/Z/10/Z from the Wellcome Trust. M.P.S. is the recipient of scholarship STE 2091/1-1 from the Deutsche Forschungsgemeinschaft. We are indebted to Jon Driver for his inspiration and Chris Kluge for help with data acquisition.

This article is freely available online through the J Neurosci Author Open Choice option.

Correspondence should be addressed to Markus Bauer, School of Psychology, University of Nottingham, Nottingham NG7 2RD, UK. E-mail: markus.bauer@gmail.com.

DOI:10.1523/JNEUROSCI.3474-13.2014

Copyright $\odot 2014$ Bauer et al.

This is an Open Access article distributed under the terms of the Creative Commons Attribution License Creative Commons Attribution 4.0 International, which permits unrestricted use, distribution and reproduction in any medium provided that the original work is properly attributed.
(Fries et al., 2001; Bauer et al., 2006; Siegel et al., 2008) is seen in several lines of experimental work. This temporal dissociation begs the question as to whether these frequency-specific effects are functionally distinct or whether they reflect the same topdown modulation that is merely expressed differentially, depending on stimulus-driven neuronal activation. It is well known that low-frequency alpha (and usually beta) oscillations are suppressed by stimulation (Pfurtscheller and Lopes da Silva, 1999; Bauer et al., 2006), whereas gamma oscillations are enhanced (Hoogenboom et al., 2006; Donner and Siegel, 2011). In other words, high- and low-frequency oscillations show different responses to afferent input, and this might be a sufficient explanation for the temporal dissociation of their attentional modulation. An alternative explanation calls on distinct top-down signals where one is inhibitory (Klimesch et al., 2007; Jensen and Mazaheri, 2010) and the other is facilitative (Womelsdorf et al., 2007). Recently, on the basis of laminar-specific spike frequency coherence in high and low frequencies in primates (Maier et al., 2010; Buffalo et al., 2011), Bastos et al. (2012) suggested that low-frequency oscillations provide predictions via feedback connections, while gamma oscillations signal the mismatch of sensory input to these predictions (the prediction error) via feedforward connections to higher-level brain regions.

Here, we investigate this proposal in two visual spatial attention experiments. The first experiment follows a "classical design," where participants had to discriminate the orientation of a static grating, a feature available from stimulus onset. In experiment 2 , the task was to detect a speed change (at a cued location) that occurred several hundred milliseconds after stimulus onset. Hence, in experiment 2 , in contrast to experiment 1 , the timing of visual stimulation and onset of the attended feature were disso- 
ciated. If the temporal profiles of alpha/beta and gamma modulations can be explained by the stimulus-related suppression of alpha modulations and induction of gamma modulations, one would expect to see the same temporal pattern of alpha and gamma modulation in both experiments. However, if alpha modulation reflected a specific expectation signal before the onset of the task-relevant stimulus feature, one would expect the alpha modulation to persist beyond stimulus onset in experiment 2 .

\section{Materials and Methods}

Participants. Eight subjects ( 3 males; mean age, 27.25 years; SD, 4.5 years) participated in experiment 1 , and 10 subjects participated in experiment 2 (5 males; mean age, 24.2 years; SD, 4.8 years). Subjects were recruited from a local database and provided informed consent for the study protocol, which was endorsed by the local ethics committee.

Task. In both experiments (Fig. 1), stimuli were presented via a digital LCD projector on a screen (height, $32 \mathrm{~cm}$; width, $42 \mathrm{~cm}$; distance from participant, $\sim 70 \mathrm{~cm}$ ) inside a magnetically shielded room using Presentation software (Neurobehavioral Systems). In both experiments, a central visual cue (arrow pointing left or right, pseudo-randomized with equal frequency of occurrence, subtending $\sim 1^{\circ}$ of visual angle) was presented for $500 \mathrm{~ms}$, instructing participants to subsequently attend to the left or right of fixation. This cue was deterministic and informed participants of the behavioral relevance of the upcoming two stimuli (bilateral gratings, either left or right relevant), rather than predicting with a certain probability the location of a stimulus. This allowed us to compare brain activity in the two hemispheres as a mere function of spatial attention, while keeping the physical stimulus identical across experimental conditions. After a variable baseline interval (1300-1700 ms in experiment 1 and 1000-1500 $\mathrm{ms}$ in experiment 2), the bilateral stimuli were presented in the left and right hemifields.

In experiment 1 (Fig. $1 A$ ), two Gabor-like patches (sinusoidal stimuli with a carrier frequency of 0.5 cycles $/{ }^{\circ}$ multiplied with a Tukey window size of $9^{\circ}$ ) were presented with a slight tilt from the vertical axis. Participants were asked to indicate the direction of the tilt with respect to the vertical axis (left index finger counter-clockwise tilt-right index finger clockwise tilt). Participants were trained on the task, and the tilt angle was adjusted using a block-wise staircase procedure (to minimize influences of lapses) that increased the tilt angle if the participant committed $>20 \%$ errors and decreased if they committed $<10 \%$ errors. This procedure familiarized the subjects with the task requirements and made the task sufficiently engaging (with performance levels at $\sim 80-90 \%$ accuracy). During the experiment, the gratings were presented at eccentricities of either $6^{\circ}$ or $8^{\circ}$ of visual angle. This factor was not of relevance to the question at hand (since MEG does not measure responses at the receptive field level), so we pooled trials across both eccentricities.

In experiment 2 (Fig. $1 B$ ), stimuli comprised concentric circles adapted from Schoffelen et al. (2005) and Hoogenboom et al. (2006) with a contracting motion (standard speed, $\sim 0.5$ cycles $/ \mathrm{s}$ ). Stimuli were always presented bilaterally at a (symmetric) eccentricity of $8^{\circ}$ of visual angle at two different contrast levels $(100 \%$ or $66 \%$, the same for both stimuli). Again, we averaged across this factor. Stimuli were modulated using a Tukey window of size $9^{\circ}$. For the full set of stimuli, a cycle was divided into 250 phases (to enable smooth motion at various speeds); that is, for the progression of one circle to the nearest inward position (spatial cycle), there were 250 frames available in total (of which only a subset was presented on successive frames). After a stimulus onset asynchrony (SOA) of $382 \mathrm{~ms}$, the speed of this motion became faster or slower at a time point selected randomly (in discrete steps of $\sim 16.6 \mathrm{~ms}$ ) until $1362 \mathrm{~ms}$ after stimulus onset, independently on either side. The speed change could therefore occur on either side at random timing, but only the cued side was task relevant. In $20 \%$ of trials, no speed change occurred on either side. Participants were asked to indicate the direction of speed change (faster or slower) by means of a button press with the left (slower) or right (faster) index finger. Participants were trained on this task with a staircase procedure, increasing the level of the speed change if their performance fell below $80 \%$, and decreasing, if it rose above $90 \%$.

\section{A Experiment 1}
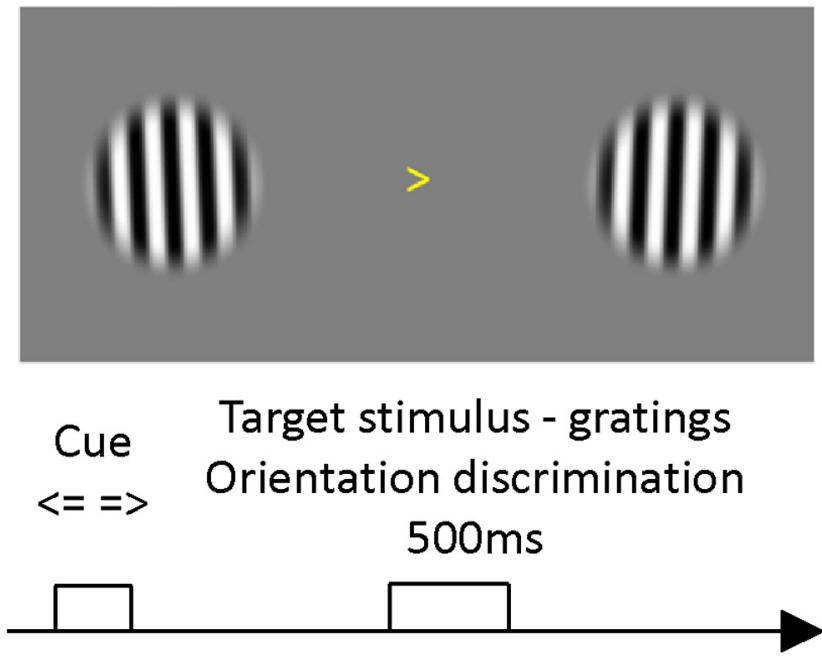

B Experiment 2
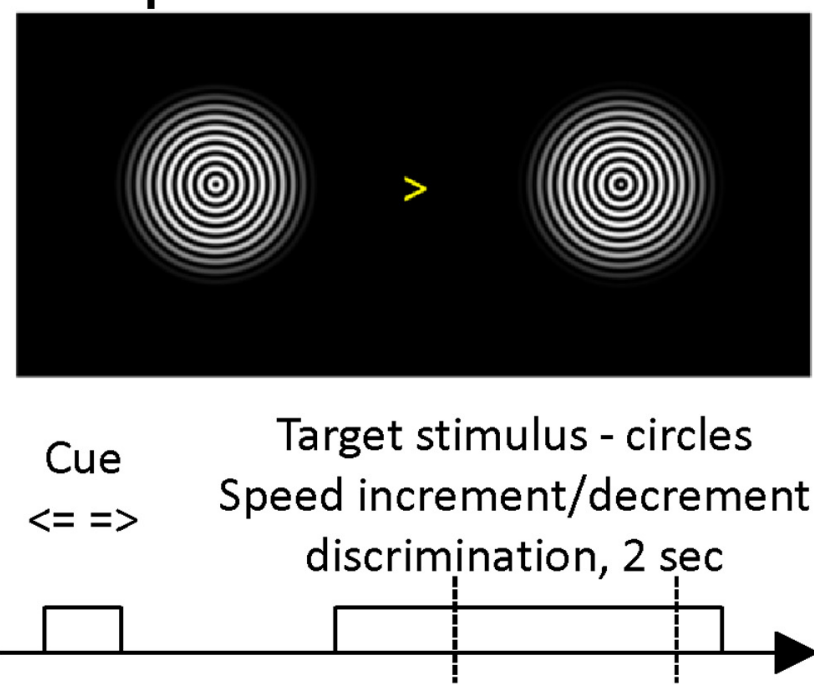

Figure 1. Experimental task. $\boldsymbol{A}$, Experiment 1 subjects had to report the deviation of a precued grating from the vertical orientation (clockwise vs counter-clockwise) by button press. The deterministic cue appeared with a variable interstimulus interval of 1300-1700 ms before the simultaneous presentation of both gratings and was followed by fixation, so the grating and cue were never presented simultaneously. $\boldsymbol{B}$, In experiment 2 , two sets of concentric gratings contracting toward the fixation point shown after a centrally presented (likewise deterministic) cue instructed participants to focus on the left or right hemifield (variable cue stimulus interstimulus interval of 1000-1500 ms). A speed change target occurred between 382 and 1362 ms following stimulus onset, and participants had to report whether the precued grating changed to a faster or slower speed.

$M E G$ recording and analysis. Data were recorded continuously from 274 axial gradiometers using a CTF Omega whole-head system at a sampling rate of $600 \mathrm{~Hz}$ in third-order gradient configuration. MEG data analysis was implemented in SPM8 (Litvak et al., 2011) and Fieldtrip (Oostenveld et al., 2011). Data preprocessing comprised the following steps: data were converted to SPM8 format and notch filtered from 49 to $51 \mathrm{~Hz}$ (on the entire unepoched dataset). Eye blinks were detected using a semiautomatic thresholding procedure, a $z$-transform of the absolute value of the 5-15 Hz bandpass-filtered Hilbert transform, with interactive threshold setting (this was done blind to the condition and for all trials of the session simultaneously). These data were then inspected for excessive artifacts, and a principal component analysis was computed over the eye-blink periods. In all participants, the first spatial component reflected the eye blink topography (an obvious "bipolar pattern" over frontal and temporal sensors). The data were then epoched: for experi- 
ment 1 (short stimulus presentation) a window of -2000 to $1200 \mathrm{~ms}$ around stimulus onset was used, whereas for experiment 2, the epoch ranged from -2000 to $2500 \mathrm{~ms}$ after stimulus onset. Eye-blink artifacts were then suppressed by removing the first principal component above using signal space projection. Finally, epochs were visually inspected (using ft_reject_visual.m), and trials with excessively high power (summed over all channels) were removed. For source analysis, individual electromagnetic forward models were constructed based either on an individual structural T1-weighted MRI (when available) or on the default MNI brain coordinates (when not). For those participants with an individual structural MRI ( 5 of 8 participants in experiment 1 and 8 of 10 participants in experiment 2), the T1 MNI brain was first warped on their individual structural image, and this transform was then applied to the (canonical) cortical surface mesh, as described in the study by Mattout et al. (2007) (see also Henson et al., 2009). For beamformer solutions, covariance matrices were calculated for the low and high frequencies. For low frequencies, the data were low-pass filtered ( $35 \mathrm{~Hz}$ cutoff frequency), and the covariance matrix was calculated over the entire epoch. The same approach was used for high frequencies, where the data were bandpass filtered at $40-150 \mathrm{~Hz}$ (data were low pass filtered during recording with a $150 \mathrm{~Hz}$ antialiasing filter). Beamformer filters were then calculated for 413 cortical vertices on a (downsampled) cortical mesh (Van Veen et al., 1997). Time series data were then projected through the resulting beamformer coefficients to produce time courses for subsequent time frequency analysis. Separate reconstruction of high-frequency responses reflects the fact that low-frequency responses require a narrower bandwidth, whereas higher frequencies have a larger bandwidth. To account for this, we used a multitaper approach for high-frequency responses (Mitra and Pesaran, 1999) that allows smoothing in both time and frequency. For low frequencies, time windows of $400 \mathrm{~ms}$ (from -1.95 to $0.8 \mathrm{~s}$ in experiment 1 , and from -1.95 to $2.5 \mathrm{~s}$ in experiment 2 , both in steps of $0.1 \mathrm{~s}$ ) were multiplied with a Hanning window and then multiplied with complex exponentials at frequencies from 2.5 to $30 \mathrm{~Hz}$ in steps of $2.5 \mathrm{~Hz}$. For the high frequencies, time windows of $400 \mathrm{~ms}$ (from -1.95 to 0.8 in experiment 1 and to 1.5 in experiment 2 , both in steps of $0.05 \mathrm{~s}$ ) were used with seven discrete prolate Slepian tapers multiplied with complex exponentials at frequencies from 40 to $150 \mathrm{~Hz}$ in steps of $5 \mathrm{~Hz}$ (with an effective smoothing of $10 \mathrm{~Hz}$ in both directions). The resulting cortical power spectra for each trial were then log transformed, and outliers (above and below 2 SDs from the mean) were removed for each timefrequency bin. Finally, condition-specific responses were summarized with statistics (mean and SEM over trials within the condition) for "attend left" and "attend right" separately. The resulting condition-specific power spectra were subtracted from baseline values (from -1.95 to $-1.85 \mathrm{~s}$ ) and smoothed using a moving average over three neighboring time-frequency bins to accommodate well known interindividual differences in induced responses (Muthukumaraswamy et al., 2009; van Pelt et al., 2012).

Statistical analysis. To quantify attentional effects, we first calculated the attentional lateralization (AL) index for condition-specific powerestimates as follows:

$$
\begin{aligned}
\operatorname{AL}(r, f, t)=\left(S(r, f, t)_{\text {attendL }}-S(r, f, t)_{\text {attendR }}\right) /(\operatorname{abs}( & \left.S(r, f, t)_{\text {attendL }}\right) \\
& \left.+\operatorname{abs}\left(S(r, f, t)_{\text {attendR }}\right)\right),
\end{aligned}
$$

where $S(r, f, t)$ is the power spectral density at a cortical vertex $r$ for frequency $f$ at time $t$ (absolute values for denominators to accommodate for rare cases where the sum of baseline-corrected values in individual bins for AttendLeft versus AttendRight gave near-zero values for denominator).

We determined the nearest homologous vertex in the left hemisphere for each vertex in the right hemisphere. We then subtracted the right $\mathrm{AL}$ values from their corresponding left hemisphere values, effectively summarizing mirror symmetrical attentional modulation (Bauer et al., $2012 \mathrm{~b}$ ). The resulting data were subjected to a cluster-based permutation test (Maris and Oostenveld, 2007) using a one-sample $t$ test (of attentional lateralization against zero) to find significant attentional effects. This procedure corrects for multiple (nonindependent) comparisons over cortical vertices, time and frequency. We performed cluster-based inference separately for the low and the high frequencies, and focused on the prestimulus period before the target and the poststimulus period. We therefore searched over $5-25 \mathrm{~Hz}$ (for low-frequency modulation effects) and $40-150 \mathrm{~Hz}$ (for high-frequency effects). The time window considered was -500 to $800 \mathrm{~ms}$ after stimulus onset (for experiment 1 ) and -500 to $1500 \mathrm{~ms}$ (for experiment 2), because our hypotheses were about the neuronal dynamics around stimulus onset and during subsequent processing.

Dependency of behavioral and physiological responses on hazard rate. To investigate the effect of expectations in experiment 2 on behavioral and electrophysiological responses, we collapsed trials over nine time bins according to the SOA between stimulus onset and the target (speed change). The cumulative probability of a speed change occurring within a certain bin (the hazard rate) as well as negative and inverse reaction time (as two different measures of behavioral response speed) were averaged, and the SD of reaction time (RT) was calculated for each bin. This revealed a large negative correlation between reaction time and the cumulative probability (both averaged in bins of trials) of $r=-0.98$ across participants, as well as a negative correlation of cumulative probability with the SD of binned reaction times $(r=-0.71)$, indicating faster and less variable responses when the stimulus change was expected. Inverse reaction time and hazard rate were also highly correlated $(r=0.9)$. Since cumulative probability and negative response time showed a rather strong linear relationship (see Fig. 3), we chose the cumulative probability as the relevant metric of stimulus expectation (in contrast to the hazard rate; Schoffelen et al., 2005).

To characterize the time courses of attentional lateralization in terms of stimulus expectation, a weighted mean of attentional lateralization in each participant was calculated from all significant vertices (grid points in the cortical mesh). The weights assigned to each vertex were the proportion of significant time-frequency bins over all time-frequency bins. To average the time courses of attentional lateralization over frequencies, we calculated again a weighted average where the time courses obtained for each frequency were weighted by the proportion of (multiple comparison corrected) significant bins for each frequency. This measure precludes biased selection of frequencies or vertices and identifies responses that survive correction for multiple comparisons, weighted by the proportion of significant effects in time and frequency bins. These individual time courses were then used for a mixed-effects ANOVA to test for differential peristimulus attentional lateralization effects in the two experiments (using prestimulus vs poststimulus times as a repeated-measures factor and experiment 1 vs 2 as a between-subject factor).

To investigate more precisely how the attentional lateralization in low and high frequencies depends on target (speed change) expectation, it was necessary to separate the trials with early and late speed changes. This is because trials with early targets would otherwise contribute to the estimation of responses induced by late targets. Therefore, we time locked the spectral estimates in each trial to the target (speed change) onset in four categories of trials, defined by the SOA between stimulus and target onset. These categories reflect different target timings and therefore specific values of the underlying hazard function that determines the strength (or precision) of expectations. We then regressed the attentional lateralization index (entire time frequency matrix from -500 to $500 \mathrm{~ms}$ around target onset) on the timing category and report the associated $t$ values (thresholded at $p<0.05$, two-tailed; see Fig. 4).

Correlation of gamma to reaction time. Finally, we conducted a control analysis to ensure that we could reproduce previously reported correlations of gamma modulation with reaction time. To this end, we split the trials of each subject into seven reaction time bins (for the same approach, see Bauer et al., 2009) and then regressed the binned response on reaction time, reporting the associated $t$ values. This analysis was performed over an average of vertices within $3 \mathrm{~cm}$ of the right calcarine sulcus. The results confirmed that stronger induced gamma is associated with faster responses (see Fig. 5).

\section{Results}

Participants in experiment 1 gave correct responses in a mean of $88.9 \%$ of the trials (SD, 9.3\%) with an average response latency of $659 \mathrm{~ms}$ (SD, $126 \mathrm{~ms}$ ). In experiment 2, participants responded with an average latency of $827 \mathrm{~ms}$ (SD, $119 \mathrm{~ms}$ ) with a mean accuracy of $80.5 \%$ (SD, 12.7\%). We had no hypotheses concerning 
the effect of the experimental conditions (or comparisons between experiments) regarding the behavioral data. We discuss the correlations of reaction times with the predictability of targets in greater detail below.

\section{Attentional modulation of low and high frequencies}

Figure 2 shows the stimulus-induced responses and the effects of spatial attention in both experiments at the cortical and source level, in Figure $2 A-F$ for the alpha/ beta band and in Figure $2 G-L$ for the gamma band. Figure 2, $A$ and $D$, shows the stimulus-induced responses (averaged $z$ values for the comparison between peristimulus time and precue baseline) in occipital cortex for experiment 1 (gratings) and experiment 2 (moving circles), respectively. Note the suppression of lowfrequency activity at stimulus onset in both experiments, as would be predicted from many previous studies (Bauer et al., 2006; Hoogenboom et al., 2006). Figure $2 B$ shows the comparison of alpha lateralization for "attend-left" - "attend-right" trials in experiment 1 . This shows the relative suppression of alpha/beta power in the hemisphere contralateral to the attended hemifield (or ipsilateral enhancement) and originates predominantly from the parieto-occipital sulcus, which is in agreement with previous findings (Fig. $2 C$; Bauer et al., 2012b). Note that this effect occurs predominantly in the period preceding the stimulus, consistent with numerous previous studies (Worden et al., 2000; Fu et al., 2001; see Discussion). Figure $2 E$ shows the attentional lateralization in experiment 2. Note that, here, attentional suppression of alpha power appears in the poststimulus period. It is also present before stimulus onset, but is less pronounced than in the poststimulus period, particularly toward the end of the trial. Figure $2 F$ shows the thresholded topography of this effect, indicating that it is more distributed; involving occipitotemporal areas, the temporoparietal junction (TPJ) and premotor areas (e.g., the frontal eye field). Crucially, in experiment 2 , the peak attentional alpha/beta modulation was seen in the poststimulus period, after stimulus onset, whereas in experiment 1 it was found predominantly in the prestimulus period (Fig. 3, time courses of both effects).

Figure 2, $G$ and J, shows the stimulusinduced gamma oscillations for both experiments. In experiment 1 (Fig. $2 G$ ), we see an increase in gamma band oscillations $\sim 500 \mathrm{~ms}$, which then return to baseline. The later lower-frequency increase is

\section{Alpha-/beta band (low frequencies)}
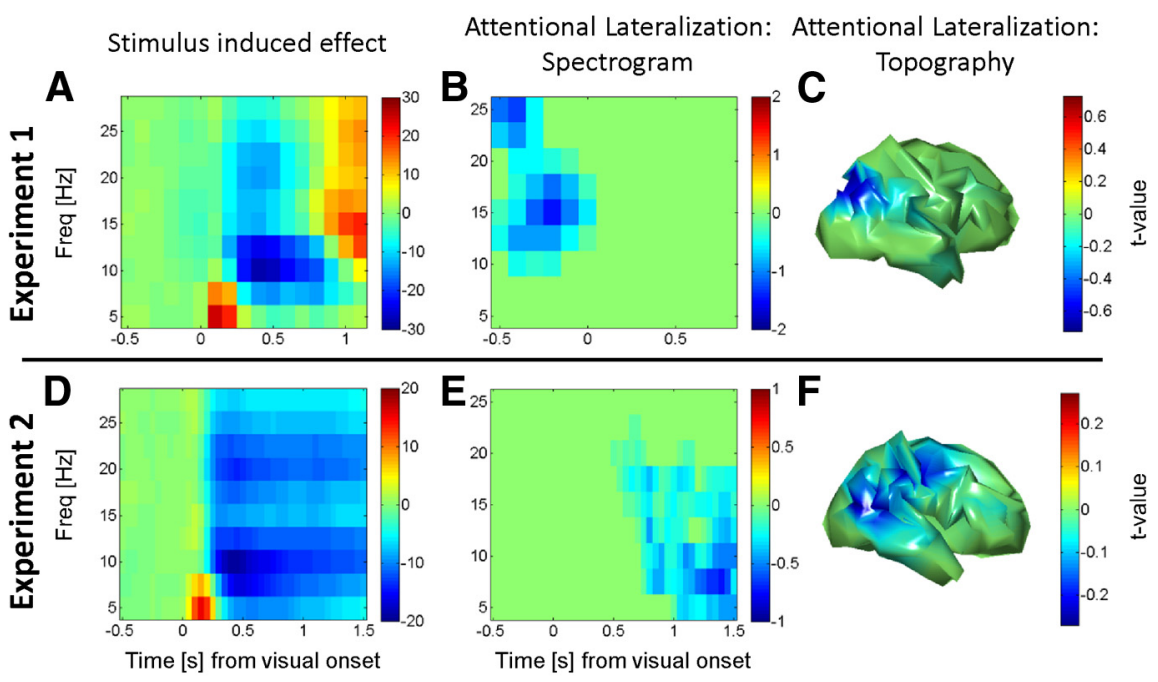

\section{Gamma-band (high frequencies)}

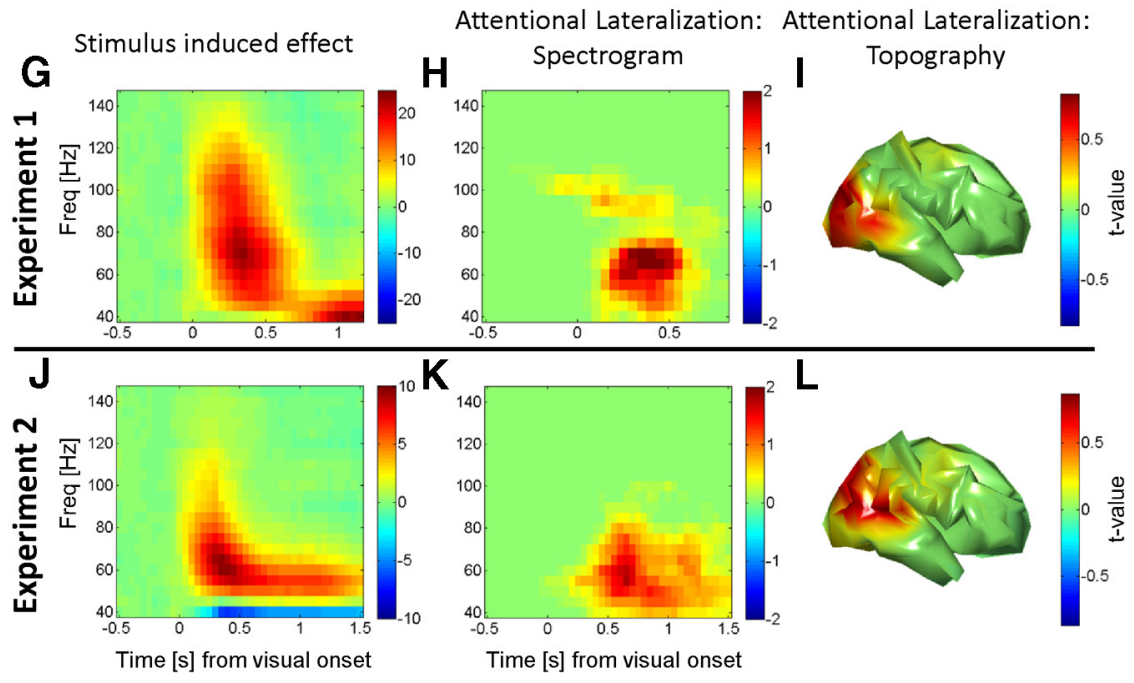

Figure 2. Stimulus- and attention-related modulation of oscillatory activity. The first column shows a time-frequency spectrum of the stimulus-induced "event-related desynchronization." The second column shows thresholded time-frequencyresolved statistical parametric maps for attentional lateralization, for combined cortical hemispheres and corrected for multiple comparisons across time, space, and frequency. The third column shows the brain topographies (hemispheric asymmetries) of these lateralized attentional effects corrected for multiple comparisons (low frequencies). $\boldsymbol{A}$, In experiment 1 , alpha and beta activity in occipital cortex are suppressed after the onset of the stimulus $(t=0)$. The grand-averaged baseline normalized response (averaged $z$ values for the within-subject comparison of peristimulus events and baseline) is shown. B, Suppression of prestimulus alpha/beta activity in the hemisphere contralateral to the attended hemifield. Note that significant attentional modulation is restricted to the prestimulus period, in the absence of visual stimulation, and while alpha oscillations are stronger. The spectra are averaged across all cortical vertices that show whole brain (hemisphere)-corrected significant modulations. C, Topography of this effect (averaged across all significant time-frequency windows) shows that the suppression of alpha-beta power is dominant in the parieto-occipital area, contralateral to the attended hemifield (combined left and right). $\boldsymbol{D}$, In experiment 2, as in $A$, alpha and beta oscillations in occipital cortex are suppressed after stimulus onset (of the moving concentric circles), showing the typical effect of stimulation on low-frequency oscillations. $\boldsymbol{E}$, Suppression of contralateral alpha-beta activity, as in $\boldsymbol{B}$, but notice here that the (cluster level-significant) attentional modulation is in the poststimulus period during strong afferent stimulation. This does not imply that there is no prestimulus modulation, but that modulation is predominant in the poststimulus period. $\boldsymbol{F}$, Topography of this effect (all significant time-frequency windows) now spreading toward more temporal areas and TPJ as well as premotor areas (high frequencies). $\mathbf{G}$, In experiment 1 , gamma activity in occipital cortex is enhanced after onset of the grating stimulus $(t=0)$. Shown is the grand-averaged baseline normalized response (averaged $z$ values for the within-subject comparison of peristimulus events and baseline). $\boldsymbol{H}$, Enhancement of poststimulus gamma activity in the hemisphere contralateral to the attended hemifield. Attentional modulation peaks in the poststimulus period. $I$, This contralateral enhancement is predominantly found in occipital and occipitotemporal cortex. J, In experiment 2 , similar to $G$, stimulus onset induces enhanced gamma oscillations, as described previously. $\boldsymbol{K}$, Likewise, contralateral gamma enhancement by spatial selective attention. There is no qualitative shift here. $\boldsymbol{L}$, Contralateral enhancement, similar to that in $I$. Freq, Frequency. 
TimeCourse Attentional Lateralization: Experiment 1

A

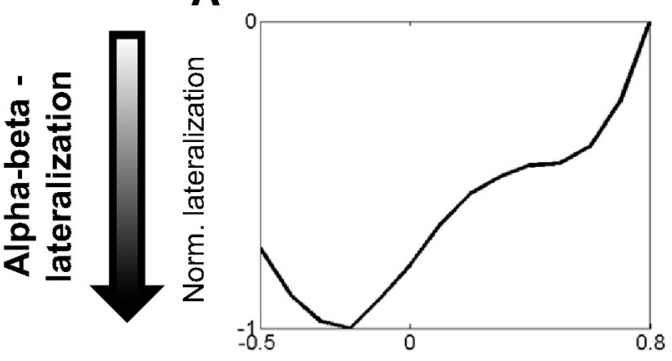

C

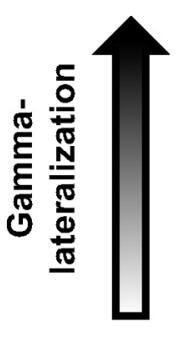

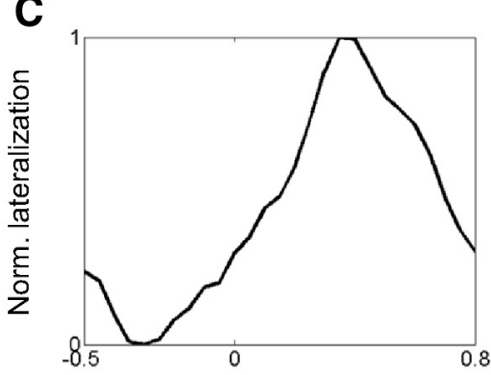

Time [s] from visual onset
B

\section{TimeCourse Attentional Lateralization: \\ Experiment 2}

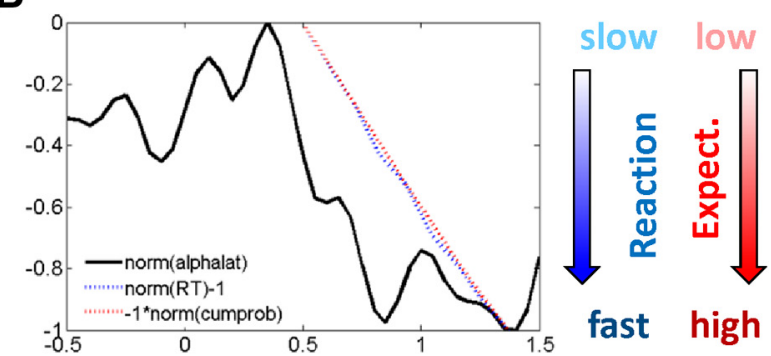

D

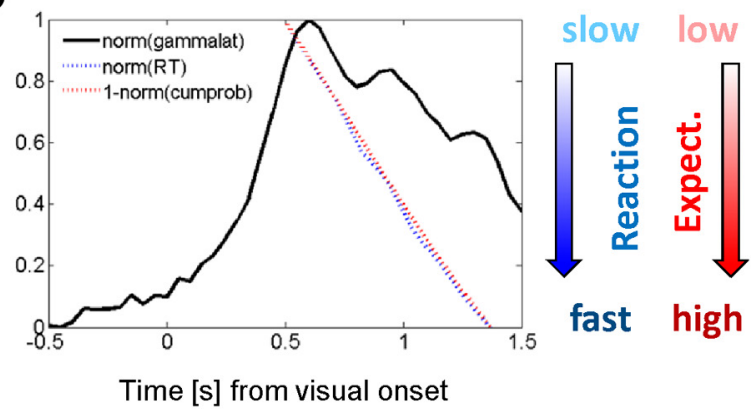

Figure 3. Time course of attentional lateralization, expectancy, and behavior. $A$, Experiment 1. The time course of the normalized lateralization index for alpha/beta-power is shown for the static gratings. Negative values indicate stronger contralateral alpha/beta-suppression (both $\boldsymbol{A}$ and $\boldsymbol{B}$ ); these are predominantly found in the pre-stimulus period and then return to baseline. $\boldsymbol{B}$, Experiment 2. Likewise, this shows the time course of normalized attentional lateralization for alpha/beta-oscillations, now for the dynamic stimuli. All measures shown in $\boldsymbol{A}$ and $\boldsymbol{B}$ are normalized to range between 0 (smallest magnitude) and -1 (greatest magnitude) for better visual comparison. For RT (blue), negative values indicate fast responses (norm[RT] -1 ) and for cumulative probability (red), negative values reflect high probability $\left(-1^{*}\right.$ norm [cumprob]). Attentional lateralization in Experiment 2 is already present in the pre-stimulus period but reaches its peak towards the end of the trial. The comparison between measures reveals that as the cumulative probability for a target increases, the magnitude of attentional alpha suppression increases and participants respond more quickly. $\boldsymbol{C}$, Experiment 1. The normalized time course of gamma-lateralization is shown. Here (both $\boldsymbol{C}$ and $\boldsymbol{D}$ ), larger positive values indicate higher magnitude of attentional gamma enhancement. $\boldsymbol{D}$, Experiment 2. Importantly, a different scaling of the $y$-axis (now from 0 to +1 ) compared to Fig. $3 \boldsymbol{B}$ is used here, for visual alignment of the measures, while the graphical slopes of RT and probability remain the same. Here, more positive lateralization values indicate greater attentional modulation and the probability is now plotted as (1-norm[cumprob]), or 'surprise'. Hence, ' 1 ' indicates low probability (high surprise) and ' 0 ' indicates high probability (low surprise). Reaction time is displayed as normalized positive RT (i.e., ' $O$ ' indicates fast responses). High gamma lateralization is therefore correlated with a state of high surprise, rather than high expectancy (contrary relationship to $\boldsymbol{B}$ ). $\boldsymbol{B}$ and $\boldsymbol{D}$ thus reveal that temporal expectancy determines the strength of alpha/beta- and gamma-lateralization, but their relationship is of opposite nature: alpha/beta correlates positively with expectancy, gamma with its complement, surprise or prediction error.

a result of the so-called beta rebound (Jensen et al., 2005; Bauer et al., 2006; Fig. 2A) that extends into higher frequencies, partly due to the spectral smoothing characteristics of the multitaper frequency analysis. In Figure 2J, we see the same for experiment 2 with the sustained motion stimulus, initially high frequencyinduced gamma oscillations that then turn into a sustained gamma band response at a slightly lower frequency. Figure $2 \mathrm{H}$ shows the attentional enhancement of gamma-band power in contralateral visual cortex for experiment 1 . The peak of this effect is seen clearly in the poststimulus period, although some sparse modulations at (and above) $100 \mathrm{~Hz}$ appear at stimulus onset. Figure 2I shows that the topography of this effect is dominated by extrastriate occipital cortex. Figure $2 K$ shows the thresholded spectrum of the attentional modulation for experiment 2 -with a similar pattern to that shown in Figure $2 \mathrm{H}$, although somewhat more sustained, given the nature of the task. Figure $2 L$ shows its topography, which again extends slightly more toward temporal areas, presumably due the nature of the speed change detection task.

Figure 3 shows the time courses of these attentional modulations. These time courses reflect weighted averages of the attentional lateralization index (i.e., the normalized power difference between hemispheres corresponding to the attended vs the unattended hemifield). These results replicate the basic finding in Figure 2: in experiment 1, the peak of the lateralized alpha-beta modulation (Fig. $3 A$ ) is in the prestimulus period, then slowly returns to baseline (and might rebound toward the end of the trial in opposite direction). Conversely, in experiment 2, while the attentional lateralization is already observable in the prestimulus period, the peak emerges toward the end of the trial, in the poststimulus period. Our main aim was to investigate whether, in a paradigm in which the task-relevant stimulus (change) occurs after stimulus onset (experiment 2), alpha/beta lateralization was observable in the later poststimulus period. Figures 2 and 3 illustrate that this is indeed the case. We nevertheless tested explicitly for a significant time shift of the dominant period of alpha lateralization in experiment 2 compared with experiment 1 (the latter reflecting the temporal pattern found in many previous studies). A $2 \times 2$ ANOVA was performed on the attentional lateralization with repeated-measures factor "time interval" (prestimulus vs poststimulus; Figs. 2, 3, time intervals) and between-subjects factor "experiment" (dynamic vs static gratings). The interaction was significant $\left(F_{(1,1,16)}=11.17, p<\right.$ $0.01)$, confirming the dissociation induced by the two experimental paradigms with respect to timing.

Relation of stimulus change probability, behavior, and physiology (experiment 2)

We separated trials into nine equally spaced bins (from 433 to $1433 \mathrm{~ms}$ ) according to the SOA of the stimulus change (target) to 
the display onset and examined the correlation between the average RT and the hazard rate for these bins. In accord with previous studies, participants appear to have tracked this probabilistic structure rather well, as reflected in a correlation of $r=0.9(p<0.01)$. The hazard rate (a conditional probability) increases relatively sharply toward the end of the trial. In contrast, the cumulative probability indicates the probability-across all trials-that a stimulus change will have occurred at any given point in poststimulus time. Due to the uniform probability distribution, this is a linear function. To make these measures easier to compare, Figure $3, B$ and $D$, shows the normalized (and sign-inverted) cumulative probability of stimulus change as a function of SOA (aligned with the time axis for the attentional lateralization) and the average reaction times for the respective time bins (individual bins are connected by line segments). These results illustrate the tight relationship between these measures $(r=-0.98$ for averaged bins and RT, $p<0.01$ ), indicating that participants learned this "probabilistic structure" and form precise expectations about the occurrence of the target (change in speed). The precision of stimulus expectancy should also be reflected in the SD of reaction times. Indeed, there was also a negative correlation between cumulative probability and the SD of reaction times $(r=$ $-0.71, p<0.01)$, confirming that strong expectations reduce not only the average response latency, but also their variance.

Figure $3 B$ shows another salient feature; namely, the fact that the strength of attentional alpha lateralization seems to follow the cumulative probability (or rather temporally precede it by a few hundred milliseconds). The correlation of alpha lateralization with cumulative probability was moderate, although significant across time bins and participants $(r=-0.25, p<0.05)$. For the gamma lateralization, the pattern is reversed (note that "maximal absolute alpha lateralization" is inverted, placing the largest magnitude of contralateral suppression at the bottom): gamma lateralization is maximal just after the earliest possible time the target can appear. With an increasing cumulative probability for target appearance, gamma lateralization is clearly reduced in amplitude $(r=-0.43, p<0.05)$, hence showing the opposite pattern with respect to the absolute strength of attentional modulation.

There is, however, one shortcoming with the above analysis: the average calculated across all trials (with the spectra giving estimates of lateralized power) includes attentional lateralization after the stimulus change has already occurred for the later time bins. To avoid this possible confound, we conducted an additional analysis in which we focused on the responses locked to the speed change. Spectral estimates of attentional lateralization were time locked to the speed change, and trials were then sorted into four categories of SOAs between stimulus and target onset. Figure $4 A$ shows the $t$ values of the regression of alpha lateralization on SOA (significant time-frequency windows marked; $p<0.05$ two-tailed, uncorrected). This takes the form of a stronger suppression of contralateral alpha lateralization when the stimulus change occurs late in the trial (i.e., is more likely to occur). Figure $4 B$ shows the result of the same regression analysis, but now for the attentional gamma modulation [note that we inverted the sign of the predictor variable for this analysis (negative SOA), which makes a direct comparison of Figs. $4 A$ (contralateral alpha decrease by attention), $B$ (contralateral gamma enhancement by attention) more intuitive, given the inverted sign of these effects]. This regression of the gamma modulation on (negative) SOA shows the opposite of the SOA effect on alpha modulation in Figure $4 A$; there is increased gamma lateralization at short SOAs, clearly peaking after the stimulus change $(\sim 150 \mathrm{~ms})$, and a negative relationship between SOA and gamma lateralization. In

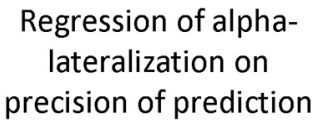

A

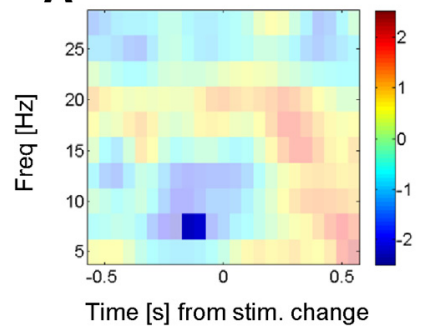

\section{Regression of gamma- lateralization on surprise (prediction error)}

B

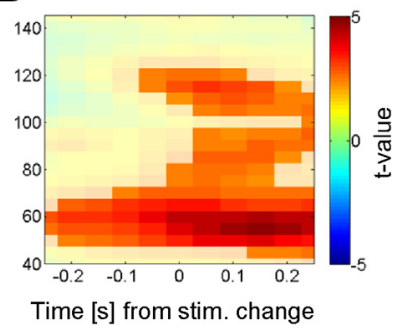

Figure 4. Oscillatory dynamics locked to the target speed change. Here we investigate how attentional lateralization develops as a function of time during the trial (and therefore cumulative probability) when considering in experiment 2 the attentional lateralization time locked to the target (speed change, time 0 ). Individual trials were binned according to the stimulus target SOA (linearly related to cumulative probability). $A$ shows the $t$ values of the regression coefficients when regressing contralateral alpha suppression on the cumulative probability of speed change. Blue values indicate stronger contralateral alpha suppression with increasing probability. Note that the temporal dynamics here follow the pattern typically observed of a prestimulus alpha lateralization, that is, attenuated after stimulus onset. This indicates that alpha lateralization indeed tracks the predictability of an upcoming target. $\boldsymbol{B}$ shows the $\boldsymbol{t}$ values of the coefficients when regressing contralateral gamma enhancement on 1-cumulative probability (i.e., the prediction error). Red values indicate larger gamma lateralization with larger prediction error. This and the poststimulus expression of this effect support the hypothesis that gamma oscillations may serve as a feedforward update signal in a predictive coding context. stim., Stimulus; Freq, frequency.

summary, the results of this analysis confirm the results of Figure 3 that more precise predictions increase the magnitude of the attentional alpha modulation and decrease gamma lateralization.

\section{High-frequency gamma responses and reaction time}

The above result implies a positive correlation between gamma lateralization and response time, when calculated over averaged binned trials sorted by target SOA. Since this result (implying stronger gamma for slow responses) is at odds with those in several reports in the literature (Womelsdorf et al., 2006; Hoogenboom et al., 2010), we characterized the relationship between induced gamma oscillations and RT when not averaging within bins sorted by SOA (which eliminates intrinsic variability in response times). The result of a regression analysis of stimulusinduced gamma oscillations in occipital cortex (ROI around the calcarine sulcus, response averaged in seven bins of trials sorted by reaction time) on reaction time per se clearly shows (Fig. 5) that our study replicates previous results by showing larger stimulus-induced gamma oscillations for faster responses.

\section{Discussion}

In summary, we found that the typical prestimulus nature of attentional alpha/beta lateralization is not simply explained by an attenuation caused by afferent drive following the onset of a sensory stimulus. In experiment 2, where the task-relevant stimulus feature was delayed with respect to stimulus onset, the alpha/beta modulation peaks during strong afferent stimulation by a high-contrast motion stimulus. Instead, our results suggest that attentional alpha/beta modulation reflects the precision of expectations about impending stimulus events (as indexed by the objective hazard function and behavior; Figs. $3 B, 4 A$ ). Thus, greater alpha modulation is seen when subjects are more certain that the event is about to occur and the modulation returns to baseline when the actual evidence is presented. Conversely, attentional lateralization of gamma os- 


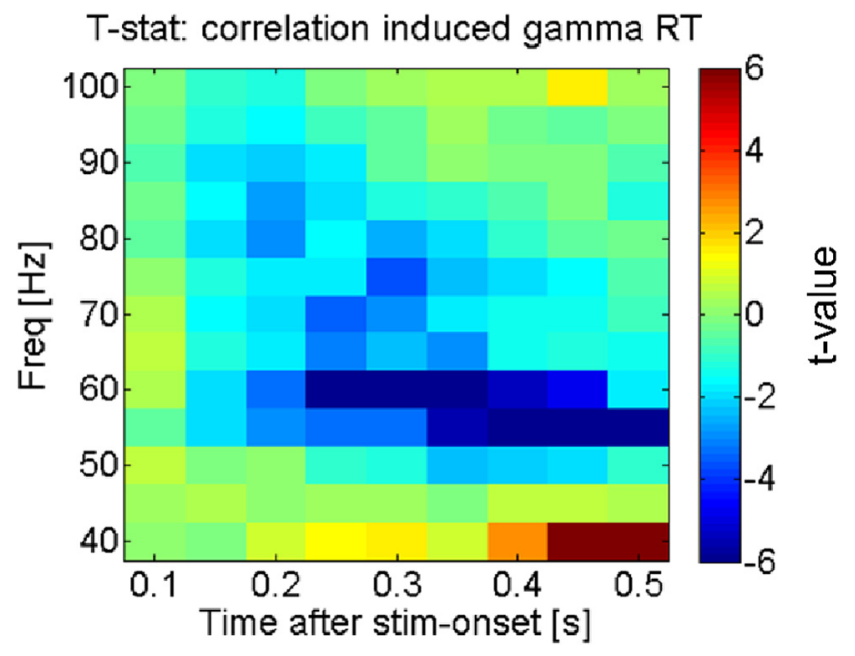

Figure 5. Correlation of RT with strength of gamma oscillations. The figure shows the $t$ statistic of the regression of occipital gamma activity on reaction times, when the entire reaction time variance is taken into account (in contrast to Fig. 3, which was averaged across trials within the same stimulus target SOA bin). Negative $t$ values indicate a negative relationship between power-spectral-density and reaction time; hence, the graph confirms that larger gamma power corresponds to shorter reaction times (faster responses) when the total variation was taken into account, confirming previous findings. stim, Stimulus; stat, statistic; Freq, frequency.

cillations is stimulus bound, and its strength depends on the mismatch between expectations and physically presented stimuli. In other words, the strength of lateralized attentional gamma modulation predominantly postdates task-relevant stimulus features and is greater when these are unpredicted (or more surprising). So far, these results are therefore consistent with the notion that prestimulus modulations of alpha activity set the context (synaptic gain) for expected stimuli, while stimulus-bound gamma responses signal prediction errors induced by stimulus changes, particularly when the stimuli are less predicted.

The fact that alpha/beta frequency modulation reflects anticipatory predictions about stimulus events has been shown in the visual (Cravo et al., 2011, 2013; Rohenkohl et al., 2012), somatosensory (van Ede et al., 2011, 2014) and motor (Schoffelen et al., 2005) domains (see also Arnal and Giraud, 2012). These data, together with our results, are therefore consistent with suggestions that low-frequency oscillations mediate feedback or descending predictions that include predictions about the precision of specific processing channels or stimulus features that establish an attentional context for subsequent sensory processing (von Stein et al., 2000; Bauer, 2008; Bastos et al., 2012), a point we discuss in greater detail below. What is new with respect to alpha oscillations in our study is the finding that this lateralized attentional modulation can be seen under strong afferent stimulation (experiment 2). This suggests that the predominantly prestimulus nature of alpha lateralization is not simply due to the absence of feedforward excitation in this period (that might subsequently attenuate this effect), but that it reflects the predictability of an upcoming event even in the presence of afferent drive. Alpha modulations by attention typically peak just before stimulus onset and then diminish when the stimulus appears. This finding was reproduced in this study (experiment 1); in the studies by Bauer (2008), Siegel et al. (2008), Green and McDonald (2010), and Bauer et al. (2012a); and in a study in the placebo condition by Bauer et al. (2012b). In the study by Bauer (2008, chapter 3 ), we present a reanalysis of data from a previous study (Bauer et al., 2006), in which no stimulus-induced attentional alpha/beta lat- eralization was reported. This reanalysis confirmed that a significant, lateralized alpha/beta modulation in somatosensory cortex was found before stimulus onset and returned to zero during the poststimulus period, then rebounding before the onset of the next stimulus. Interestingly, there was no lateralization after the onset (and during the processing) of the tactile stimulus. This finding has very recently been confirmed, using very similar stimuli, by van Ede et al. (2014). Fries et al., 2008 showed (with invasive recordings from monkey $\mathrm{V} 4$ in a speed change detection task) that the alpha/beta attentional modulation was dominant in the prestimulus period (their Fig. 5) and much less pronounced in the poststimulus period (their Fig. 7). Hence, there is clear evidence that the peak of alpha/beta lateralization is typically found prestimulus, and that the peak gamma lateralization emerges in the poststimulus period.

With respect to the attentional modulation of gamma oscillations, our findings are more controversial. A previous study, using invasive recordings and intra-areal and interareal measures of synchronization in cats (von Stein et al., 2000), reports that lowfrequency oscillations reflect the strength of a prediction signal and gamma oscillations reflect a prediction error. Hence, these results are similar to our results, which are based on intra-areal synchronization measured with MEG. A study by Lima et al. (2011) specifically investigated the effect of expectation on stimulus-induced gamma oscillations in two monkeys using tasks where the stimulus could occur at either of two discrete time points, cued or uncued, with different probabilities. In one monkey, Lima et al. (2011) found a significant reaction time effect, but both monkeys showed increased stimulus-induced gamma oscillations with higher expectations, to some extent contrary to our findings.

There are several differences between the study by Lima et al. (2011) and ours. First, we looked at the impact of a spatially selective top-down signal on occipital activity, indexed by lateralized responses, whereas they described a global stimulation effect. Second, in our study, human subjects performed a task with a continuous probability distribution. The fact that we observe a strong correlation between cumulative probability (expectation) and average binned response time of $r=-0.98$ across subjects ( $r=-0.71$ for the SD of the response time) shows that human observers form and use their subjective precision or confidence in their predictions. Schoffelen et al. (2005) used a task similar to the one we used (in fact, our experiment 2 is a modification of their study) and showed that, at least in the motor system, corticospinal coherence in the gamma range showed a positive correlation with the hazard function, similar to the findings of Lima et al. (2011). We did not examine stimulus-induced responses for two reasons. First, our focus was on the lateralized attentional effects on alpha/beta and gamma oscillations. Second, characterizing stimulus-induced responses to a stimulus change is confounded by adaptation to the stimulus per se. Surprise-related effects on stimulus-induced gamma oscillations may be better examined using separate presentations of stimuli following a probability distribution, as opposed to predictable and unpredictable changes in an ongoing stimulus. The literature on the relation of gamma oscillations to stimulus expectations is currently based on different measures of neuronal activity; nevertheless, there seems to be an interesting heterogeneity in the relationship between gamma oscillations and the strength of predictions, which we discuss in a more principled way below.

In the context of predictive coding, it has recently been proposed that low-frequency oscillations provide prediction signals via descending (feedback) connections to a lower level areas, 
whereas gamma oscillations (reflecting feedforward propagation of neuronal activity) convey the prediction error encoded by superficial pyramidal cells (Bastos et al., 2012), consistent with our own findings and those of von Stein et al., 2000. However, the relation among predictability, attention, and the expected strength of a feedforward signal on downstream neurons is more intricate. Under generalized predictive coding (Feldman and Friston, 2010; Brown and Friston, 2013), attention is mechanistically conceptualized as the top-down regulation of the precision (inverse variance) of sensory signals through synaptic gain modulation and is typically thought to follow the anticipation (or expectation) of behaviorally relevant stimulus events. It is assumed that while predictions of a stimulus attenuate its feedforward imprint by inhibitory or subtractive effects on prediction error units in superficial layers (that are thought to be the predominant source of MEG signals), the predictability of a behaviorally relevant stimulus can also augment synaptic gain in superficial layers that may reverse the suppressive effects of the prediction per se. This gain control corresponds to an encoding of precision or confidence in prediction errors that boosts their influence on processing in the next level of the cortical hierarchy (Feldman and Friston, 2010; Brown and Friston, 2013). The ensuing changes in the membrane potential from the synaptic gain control is supposed to change the temporal properties of the neurons and lead to a shift from low frequencies to higher frequencies (Chawla et al., 1999), hence boosting gamma oscillations (reflecting the prediction error) at the expense of alpha oscillations, as has been regularly observed (Hoogenboom et al., 2006; Potes et al., 2014).

Hence, there are two mechanisms with antagonistic effects on gamma oscillations in operation, as follows: (1) top-down predictions of a stimulus event (as in classic predictive coding schemes) are proposed to suppress the prediction errors per se and therefore, according to our hypotheses (Bastos et al., 2012), gamma oscillations; and (2) attentional synaptic gain regulation can boost the efficacy of prediction errors. The hypothesized interactions between both mechanisms (suppressive effects of prediction and attentional enhancement through gain modulation) have recently been supported in two studies. A study by Jiang et al. (2013) found that attention can boost the prediction error, such that the difference between the neuronal responses to expected and unexpected stimuli is amplified when these are attended. Kok et al. (2012) have found that attention can override the suppressive effects of top-down prediction signals, such that expected stimuli that are attended yield the largest responses in early sensory cortex. Results like those by Lima et al. (2011) and Schoffelen et al. (2005) can be interpreted as manifestations of the second mechanism we have described above (gain enhancement), whereas the suppressive effects seem less effective there. Schoffelen et al. (2005) focused on motor gamma oscillations where one would not expect a suppressive effect in the first place, while the discrete timing in a relatively long interval in the study by Lima et al. (2011) may similarly diminish the suppression of prediction errors.

In our study, attention was operationalized through the manipulation of the behavioral relevance of two simultaneously presented bilateral stimuli with a deterministic cue. Hence, only one side was of behavioral relevance and therefore the effect of target prediction should be dominant in the hemisphere contralateral to the precued hemifield. The predictive coding perspective allows us to partition cortical responses into two components. The first corresponds to (prestimulus) activity due to anticipatory processing, while the second (poststimulus) response is induced by a target when it appears. In simple terms, the prestimulus activity can only reflect the confidence or precision that a target will appear in a future. We assumed that this is reflected in the desynchronization of alpha/beta activity, such that increasing precision or anticipation of an impending target increases cortical excitability and suppresses (desynchronizes) alpha activity. The second component is associated with the generation of prediction errors during the processing of the stimulus, which we associate with the amplitude of induced gamma responses. With this dissociation in mind, one would hypothesize that a target that was anticipated (later in peristimulus time) would be preceded by a desynchronization of alpha/beta power and a subsequent reduction in gamma activity, as prediction errors are explained away more efficiently. These dissociable effects reflect the increasing precision or confidence that a target will appear and the suppression of prediction errors when the target appears, respectively. These hazard rate or anticipation-dependent effects of the lateralized gamma oscillations are exactly what we observed. Nevertheless, in the light of the diversity of findings in the literature summarized here, we appreciate that more research is needed to complete our understanding of the specific interactions between suppressive and gain-enhancing effects of topdown influences within the context of predictive coding.

Furthermore, with respect to the systems generating top-down predictions of stimulus content and precision, the theoretical literature focuses on canonical microcircuits in a hierarchical network; however, dedicated brain structures have been associated with functions related to goal-directed attentional selection and stimulusdriven adaptive responses. For example, it is possible that the same mechanisms are implicated in the volitional control of selective attention and subjective precision (Corbetta and Shulman, 2002; Vossel et al., 2012). These studies suggest (dorsal) prefrontal and parietal sources of descending gain control that increase with stimulus anticipation. Conversely, if the stimulus appears relatively unexpectedly, the ventral system may boost forward propagation of unexpected stimulus changes through the hierarchy to enable a (relatively) rapid response, and this may then be reflected in the poststimulus augmentation of gamma oscillations.

Thus, we have shown that lateralized attentional alpha modulation may reflect a (prestimulus) top-down precision or gain control that persists until the anticipated event occurs and correlates with the subjective confidence or precision of the anticipated sensory signals. Furthermore, our results give support to the idea that (poststimulus) gamma oscillations are implicated in forwarding prediction errors, and their strength may reflect the degree of surprise induced by predicted sensory information rather than simply reflecting the state of neuronal excitation driven by afferent stimulation.

\section{References}

Arnal LH, Giraud AL (2012) Cortical oscillations and sensory predictions. Trends Cogn Sci 16:390-398. CrossRef Medline

Bastos AM, Usrey WM, Adams RA, Mangun GR, Fries P, Friston KJ (2012) Canonical microcircuits for predictive coding. Neuron 76:695-711. CrossRef Medline

Bauer M (2008) Functional roles of rhythmic neuronal activity in the human visual and somatosensory system. PhD Thesis. F.C. Donders Centre for Cognitive Neuroimaging, Radboud University: Nijmegen, The Netherlands.

Bauer M, Oostenveld R, Peeters M, Fries P (2006) Tactile spatial attention enhances gamma-band activity in somatosensory cortex and reduces lowfrequency activity in parieto-occipital areas. J Neurosci 26:490-501. CrossRef Medline

Bauer M, Oostenveld R, Fries P (2009) Tactile stimulation accelerates behavioral responses to visual stimuli through enhancement of occipital gamma-band activity. Vision Res 49:931-942. CrossRef Medline 
Bauer M, Kennett S, Driver J (2012a) Attentional selection of location and modality in vision and touch modulates low-frequency activity in associated sensory cortices. J Neurophysiol 107:2342-2351. CrossRef Medline

Bauer M, Kluge C, Bach D, Bradbury D, Heinze HJ, Dolan RJ, Driver J (2012b) Cholinergic enhancement of visual attention and neural oscillations in the human brain. Curr Biol 22:397-402. CrossRef Medline

Brown HR, Friston KJ (2013) The functional anatomy of attention: a DCM study. Front Hum Neurosci 7:784. CrossRef Medline

Buffalo EA, Fries P, Landman R, Buschman TJ, Desimone R (2011) Laminar differences in gamma and alpha coherence in the ventral stream. Proc Natl Acad Sci U S A 108:11262-11267. CrossRef Medline

Carrasco M (2011) Visual attention: the past 25 years. Vision Res 51:14841525. CrossRef Medline

Chawla D, Lumer ED, Friston KJ (1999) The relationship between synchronization among neuronal populations and their mean activity levels. Neural Comput 11:1389-1411. CrossRef Medline

Corbetta M, Shulman GL (2002) Control of goal-directed and stimulusdriven attention in the brain. Nat Rev Neurosci 3:201-215. CrossRef Medline

Cravo AM, Rohenkohl G, Wyart V, Nobre AC (2011) Endogenous modulation of low frequency oscillations by temporal expectations. J Neurophysiol 106:2964-2972. CrossRef Medline

Cravo AM, Rohenkohl G, Wyart V, Nobre AC (2013) Temporal expectation enhances contrast sensitivity by phase entrainment of low-frequency oscillations in visual cortex. J Neurosci 33:4002-4010. CrossRef Medline

Donner TH, Siegel M (2011) A framework for local cortical oscillation patterns. Trends Cogn Sci 15:191-199. CrossRef Medline

Feldman H, Friston KJ (2010) Attention, uncertainty, and free-energy. Front Hum Neurosci 4:215. CrossRef Medline

Fries P, Reynolds JH, Rorie AE, Desimone R (2001) Modulation of oscillatory neuronal synchronization by selective visual attention. Science 291: 1560-1563. CrossRef Medline

Fries P, WomelsdorfT, Oostenveld R, Desimone R (2008) The effects of visual stimulation and selective visual attention on rhythmic neuronal synchronization in macaque area V4. J Neurosci 28:4823-4835. CrossRef Medline

Friston K (2005) A theory of cortical responses. Philos Trans R Soc Lond B Biol Sci 360:815-836. CrossRef Medline

Fu KM, Foxe JJ, Murray MM, Higgins BA, Javitt DC, Schroeder CE (2001) Attention-dependent suppression of distracter visual input can be crossmodally cued as indexed by anticipatory parieto-occipital alpha-band oscillations. Brain Res 12:145-152.

Green JJ, McDonald JJ (2010) The role of temporal predictability in the anticipatory biasing of sensory cortex during visuospatial shifts of attention. Psychophysiology 47:1057-1065. CrossRef Medline

Henson RN, Mattout J, Phillips C, Friston KJ (2009) Selecting forward models for MEG source-reconstruction using model-evidence. Neuroimage 46:168-176. CrossRef Medline

Hoogenboom N, Schoffelen JM, Oostenveld R, Parkes LM, Fries P (2006) Localizing human visual gamma-band activity in frequency, time and space. Neuroimage 29:764-773. CrossRef Medline

Hoogenboom N, Schoffelen JM, Oostenveld R, Fries P (2010) Visually induced gamma-band activity predicts speed of change detection in humans. Neuroimage 51:1162-1167. CrossRef Medline

Jensen O, Mazaheri A (2010) Shaping functional architecture by oscillatory alpha activity: gating by inhibition. Front Hum Neurosci 4:186. CrossRef Medline

Jensen O, Goel P, Kopell N, Pohja M, Hari R, Ermentrout B (2005) On the human sensorimotor-cortex beta rhythm: sources and modeling. Neuroimage 26:347-355. CrossRef Medline

Jiang J, Summerfield C, Egner T (2013) Attention sharpens the distinction between expected and unexpected percepts in the visual brain. J Neurosci 33:18438-18447. CrossRef Medline

Klimesch W, Sauseng P, Hanslmayr S (2007) EEG alpha oscillations: the inhibition-timing hypothesis. Brain Res Rev 53:63-88. CrossRef Medline

Kok P, Rahnev D, Jehee JF, Lau HC, de Lange FP (2012) Attention reverses the effect of prediction in silencing sensory signals. Cereb Cortex 22: 2197-2206. CrossRef Medline

Lima B, Singer W, Neuenschwander S (2011) Gamma responses correlate with temporal expectation in monkey primary visual cortex. J Neurosci 31:15919-15931. CrossRef Medline

Litvak V, Mattout J, Kiebel S, Phillips C, Henson R, Kilner J, Barnes G,
Oostenveld R, Daunizeau J, Flandin G, Penny W, Friston K (2011) EEG and MEG data analysis in SPM8. Comput Intell Neurosci 2011:852961. CrossRef Medline

Luck SJ, Woodman GF, Vogel EK (2000) Event-related potential studies of attention. Trends Cogn Sci 4:432-440. CrossRef Medline

Maier A, Adams GK, Aura C, Leopold DA (2010) Distinct superficial and deep laminar domains of activity in the visual cortex during rest and stimulation. Front Syst Neurosci 4:31. CrossRef Medline

Maris E, Oostenveld R (2007) Nonparametric statistical testing of EEG- and MEG-data. J Neurosci Methods 164:177-190. CrossRef Medline

Mattout J, Henson RN, Friston KJ (2007) Canonical source reconstruction for MEG. Comput Intell Neurosci 67613. CrossRef Medline

Mazaheri A, van Schouwenburg MR, Dimitrijevic A, Denys D, Cools R, Jensen O (2014) Region-specific modulations in oscillatory alpha activity serve to facilitate processing in the visual and auditory modalities. Neuroimage 87:356-362. CrossRef Medline

Mitra PP, Pesaran B (1999) Analysis of dynamic brain imaging data. Biophys J 76:691-708. CrossRef Medline

Muthukumaraswamy SD, Edden RA, Jones DK, Swettenham JB, Singh KD (2009) Resting GABA concentration predicts peak gamma frequency and fMRI amplitude in response to visual stimulation in humans. Proc Natl Acad Sci U S A 106:8356-8361. CrossRef Medline

Oostenveld R, Fries P, Maris E, Schoffelen JM (2011) FieldTrip: open source software for advanced analysis of MEG, EEG, and invasive electrophysiological data. Comput Intell Neurosci 2011:156869. CrossRef Medline

Pfurtscheller G, Lopes da Silva FH (1999) Event-related EEG/MEG synchronization and desynchronization: basic principles. Clin Neurophysiol 110:1842-1857. CrossRef Medline

Potes C, Brunner P, Gunduz A, Knight RT, Schalk G (2014) Spatial and temporal relationships of electrocorticographic alpha and gamma activity during auditory processing. Neuroimage 97:188-195. CrossRef Medline

Rohenkohl G, Cravo AM, Wyart V, Nobre AC (2012) Temporal expectation improves the quality of sensory information. J Neurosci 32:8424-8428. CrossRef Medline

Schoffelen JM, Oostenveld R, Fries P (2005) Neuronal coherence as a mechanism of effective corticospinal interaction. Science 308:111-113. CrossRef Medline

Siegel M, Donner TH, Oostenveld R, Fries P, Engel AK (2008) Neuronal synchronization along the dorsal visual pathway reflects the focus of spatial attention. Neuron 60:709-719. CrossRef Medline

van Ede F, de Lange F, Jensen O, Maris E (2011) Orienting attention to an upcoming tactile event involves a spatially and temporally specific modulation of sensorimotor alpha- and beta-band oscillations. J Neurosci 31:2016-2024. CrossRef Medline

van Ede F, Szebényi S, Maris E (2014) Attentional modulations of somatosensory alpha, beta and gamma oscillations dissociate between anticipation and stimulus processing. Neuroimage 97:134-141. CrossRef Medline

van Pelt S, Boomsma DI, Fries P (2012) Magnetoencephalography in twins reveals a strong genetic determination of the peak frequency of visually induced gamma-band synchronization. J Neurosci 32:3388-3392. CrossRef Medline

Van Veen BD, van Drongelen W, Yuchtman M, Suzuki A (1997) Localization of brain electrical activity via linearly constrained minimum variance spatial filtering. IEEE Trans Biomed Eng 44:867-880. CrossRef Medline

von Stein A, Chiang C, König P (2000) Top-down processing mediated by interareal synchronization. Proc Natl Acad Sci U S A 97:14748-14753. CrossRef Medline

Vossel S, Weidner R, Driver J, Friston KJ, Fink GR (2012) Deconstructing the architecture of dorsal and ventral attention systems with dynamic causal modeling. J Neurosci 32:10637-10648. CrossRef Medline

Womelsdorf T, Fries P, Mitra PP, Desimone R (2005) Gamma-band synchronization in visual cortex predicts speed of change detection. Nature 439:733-736. CrossRef Medline

Womelsdorf T, Schoffelen JM, Oostenveld R, Singer W, Desimone R, Engel AK, Fries P (2007) Modulation of neuronal interactions through neuronal synchronization. [see comment]. Science 316:1609-1612. CrossRef Medline

Worden MS, Foxe JJ, Wang N, Simpson GV (2000) Anticipatory biasing of visuospatial attention indexed by retinotopically specific alpha-band electroencephalography increases over occipital cortex. J Neurosci 20:RC63. Medline 